\title{
Caracterización morfoagronómica de genotipos de piña (Ananas spp.) en un suelo de terraza alta de Villavicencio
}

\author{
Morphoagronomic characterisation of pineapple (Ananas spp.) \\ genotypes in high-terrace soil near Villavicencio
}

\author{
Caracterização morfoagronômica de genótipos de abacaxi (Ananas \\ spp.) em um solo de planalto de Villavicencio.
}

Hernando Delgado-Huertas ${ }^{1}$; Laura Arango-Wiesner ${ }^{2}$

1 Ingeniero Agrónomo, MSc, Programa de Ingeniería Agronómica, Grupo de Estudio ISAF. Innovación en Sistemas Agrícolas y Forestales. Facultad de Ciencias Agropecuarias y Recursos Naturales, Universidad de los Llanos

2 Ingeniera Agrónoma, MSc, Centro de Investigación La Libertad, Corporación Colombiana de Investigación Agropecuaria - CORPOICA, Villavicencio, Colombia.

Email: hdelgado@unillanos.edu.co

Recibido: septiembre 13 de $2013 \quad$ Aceptado: septiembre 24 de 2015

\begin{abstract}
Resumen
Colombia es centro de diversidad genética de la piña. Se prevé expansión de áreas comerciales del cultivo en los Llanos Orientales. Para el mejoramiento genético de la piña, es prioritario ampliar su base genética. Mediante caracterización y evaluación de recursos genéticos, se identifican caracteres potencialmente útiles para el mejoramiento. El objetivo fue evaluar la variabilidad genética de una colección de trabajo de germoplasma de piña del C. I. La Libertad de CORPOICA en Villavicencio (Colombia). Se aplicaron descriptores del IPGRI a cinco plantas al azar de cada uno de ocho genotipos establecidos en un suelo ácido de sabana, seis de la especie comestible Ananas comosus var. comosus y dos ornamentales $A$. comosus spp. Los datos de quince descriptores cuantitativos, vegetativos y de fruto, de cuatro de los genotipos: el híbrido MD-2, los cultivares comerciales Cayena Lisa y Mayanéz y el cultivar nativo Mitú, fueron analizados mediante comparación de medias y análisis de componentes principales. Se encontró una mayor variabilidad en los descriptores vegetativos que en los de fruto, siendo que altura de planta (ALPL); ancho de la hoja D (AHD) y peso de la hoja D (PHD); longitud del pedúnculo (LPED) y longitud del fruto (LFR), tuvieron mayor varianza explicada y capacidad discriminatoria entre genotipos. En descriptores cualitativos, se destacó una alta variabilidad en la forma de distribución de las espinas en márgenes de hojas. Se identificaron diferencias y semejanzas morfológicas entre estos genotipos. Es importante continuar la introducción, caracterización y evaluación agronómica de otros cultivares nativos, como base para proyectos de desarrollo de nuevo germoplasma mejorado o para su uso directo en pequeña producción orgánica.
\end{abstract}

Palabras clave: piña, descriptores, variabilidad genética.

\begin{abstract}
Colombia is a centre for the pineapple's genetic diversity. Commercial crop areas are expected to expand on Colombia's eastern plains (the Llanos Orientales). The pineapple's genetic base must be expanded for its genetic improvement; potentially useful characters/features for improvement can be identified by characterising and evaluating genetic resources.
\end{abstract}


This work was thus aimed at evaluating the genetic variability of a pineapple germplasm collection kept at CORPOICA's La Libertad research centre in Villavicencio (Colombia, 336 masl). International Plant Genetic Resources Institute (IPGRI) descriptors were used with five randomly chosen plants from each of eight genotypes established in an acid savannah soil: six from the edible species Ananas comosus var. comosus and two from the ornamental species A. comosus spp. The data regarding fifteen quantitative, vegetative and fruit descriptors for four of the genotypes (the MD-2 commercial hybrid, the Cayena Lisa and Mayanéz commercial cultivars and the Mitú native cultivar) were analysed by comparing means and principal component analysis. Greater variability was found regarding the vegetative descriptors than the fruit ones, plant height $(\mathrm{PH})$, leaf width $(\mathrm{LW})$, leaf weight D (LWD), peduncle length $(\mathrm{PL})$ and fruit length $(\mathrm{FL})$ having greater explained variance and discriminatory power between genotypes. Regarding qualitative descriptors, it is worth highlighting the great variability concerning how thorns were distributed on leaf margins. Morphological differences and similarities between these genotypes were identified. The introduction, characterisation and agronomic evaluation of other native cultivars must be continued as the basis for projects aimed at developing new improved germplasm or for direct use in small-scale organic production.

Key words: pineapple, descriptor, genetic variability.

\section{Resumo}

A Colômbia é centro de diversidade genética de abacaxi. A expansão das áreas comerciais de cultivo é esperada nas planícies orientais. Para o melhoramento genético do abacaxizeiro, é uma prioridade ampliar sua base genética. Pela caracterização e avaliação de recursos genéticos, potencialmente úteis para a melhora são identifica as caracteres. O objetivo foi avaliar a variabilidade genética de uma coleção de abacaxi do germoplasma C. I. Liberdade de CORPOICA em Villavicencio (Colômbia). foram aplicados descritores IBQGR aleatoriamente a cinco plantas dos oito genótipos estabelecidos em um solo ácido de cerrado, seis da especie Ananas comosus var comosus e dois ornamentais Ananas comosus spp. Os dados de quinze descritores quantitativos, vegetativa e de frutas, de quatro genótipos: o híbrido MD-2, cultivares comerciais Cayena Lisa e Mayanéz e o cultivar nativa Mitu, foram analisados por comparação de médias e análise de componentes principais. Foi encontrada maior variabilidade nos descritores vegetativos que nas frutas, sendo que a altura da planta (ALPL); largura da lâmina D (AHD) e peso da folha D (PHD); comprimento do pedúnculo (LPED) e comprimento do fruto (LFR), teve variação explicada e capacidade de discriminar entre os genótipos. Em descritores qualitativos, houve alta variabilidade na forma de distribuição de espinhos sobre as margens da folha. Foram identificadas diferenças e semelhanças morfológicas entre estes genótipos. É importante continuar a introdução, caracterização e avaliação agronômica de outras cultivares nativas como base para projetos de desenvolvimento de novo germoplasma melhorado ou para utilização directa na pequena produção orgânica.

Palavras-chave: abacaxi, descritores, variabilidade genética.

\section{Introducción}

La piña (Ananas comosus var. comosus) es una valiosa contribución de las civilizaciones precolombinas a la horticultura del mundo y una de las frutas tropicales más importantes (Coppens d'Eeckenbrugge y Duval, 2009; Coppens d'Eeckenbrugge et al., 2011; Zhang et al., 2014). Fue vista por primera vez por los europeos cuando Colón desembarcó en la isla habitada que llamó Guadalupe el 04 de noviembre 1493, durante su segundo viaje al Nuevo Mundo (Botella y Smith, 2008). Por su aroma, sabor característico y exuberante apariencia, fue llamado el "Rey de los frutos coloniales" y se cultiva y consume en los cinco continentes (Crestani et al., 2010). Al momento de la conquista, ya era cultivada desde muchos siglos antes, por los indígenas nativos en todas las tierras bajas neotropicales de Mesoamérica y las Antillas, hacia el sur hasta el Paraguay, en los valles húmedos de la costa de Perú y ya habían desarrollado cultivares específicos para las laderas andinas, que son hoy todavía importantes en Colombia, Venezuela y Perú (Clement et al., 2010).
Existen dos centros para la diversificación genética de A. comosus var. comosus, uno primario en las Guyanas, con participación de los clones en las diferentes fases de domesticación, y uno secundario en la Amazonia Occidental/precordillera (a lo largo del alto Amazonas, cerca de la triple frontera entre Colombia, Perú y Brasil, y en el bajo Río Negro), con la recombinación sexual restringida al germoplasma domesticado entre las variedades de frutos grandes, las mutaciones somáticas y selección clonal, ocurridas hace más de 3500 años, como se deduce por la distribución de determinados tipos de márgenes de hojas (Coppens d'Eeckenbrugge y Duval, 2009; Coppens d'Eeckenbrugge et al., 2011).

Inicialmente se dividieron las variedades de piña cultivada en tres grupos: "Cayena", "Queen" y "Spanish", con base en la morfología de la hoja, espinas, color de las flores, forma y tamaño del fruto. Py (1970), adicionó los grupos "Pernambuco" y "Maipure". Duval et al,. (1997), coincidieron que sí bien esta clasificación era apropiada para propósitos prácticos, no estaba claramente definida por sus bases genéticas. Además, 
muchas variedades de uso local, no encajaban en los grupos.

Noyer et al., (1997), mediante RFLP en ADN ribosómi$\mathrm{CO}$, encontraron heterogeneidad importante del género Ananas e identificaron seis grupos. Posteriormente, Duval et al., (2000) estudiaron con RFLP la variabilidad genética en el género Ananas y Pseudoananás en 313 genotipos del banco de germoplasma del CIRAD FLHOR-Brasil, encontrando una alta variabilidad entre genotipos. Tapia et al., (2005), probaron 29 iniciadores ISSR y 20 decámeros RAPD en 12 materiales de Ananas comosus, uno de $A$. ananasoides y uno de $A$. bracteatus del Banco de Germoplasma del Campo Experimental del Papaloapán, Veracruz-México. Los agrupamientos generados por las dos metodologías fueron similares, agrupando inicialmente a los materiales más emparentados y con características morfológicas similares, pertenecientes a $A$. comosus, y finalmente los materiales menos emparentados $A$. ananasoides y A. bracteatus. Estos agrupamientos difirieron del obtenido mediante datos morfológicos, sobre todo en los materiales de A. comosus. La variación genética encontrada en $A$. comosus se atribuyó principalmente a la autoincompatibilidad de la especie, fenómeno que ha favorecido los cruzamientos y ha conducido a un alto nivel de heterocigosis, así como a factores ambientales (Py et al., 1984), la tasa de mutación y la variación somaclonal.

Con base en morfología tradicional, la clasificación de la piña se ha perfeccionado, y de dos géneros y ocho especies iniciales, se redujo a dos especies, $A$. macrodontes y $A$. comosus, y cinco variedades botánicas de A. comosus, vars. comosus, ananassoides, parguazensis, erectifolius y bracteatus, correspondiendo la piña para producción de fruta a $A$. comosus var. comosus. Las pruebas basadas en marcadores moleculares confirman esta clasificación revisada (Coppens d'Eeckenbrugge y Leal, 2003; Coppens d'Eeckenbrugge y Duval, 2009; Zhang et al., 2014). No obstante la relativa sencillez actual de la diversidad de la piña, su evolución ha sido caótica, con una inflación de sinónimos, basada inicialmente en el conocimiento de un puñado de cultivares de la piña comestible y, más tarde, reinterpretaciones de formas de menor importancia económica, pobremente descritas. Además, la naturaleza clonal de la mayoría de los materiales observados exageró la importancia de la, aparentemente estable, diversidad morfológica (Coppens d'Eeckenbrugge, 2014).

En la piña, la homogeneidad está garantizada por su propagación vegetativa, por lo que todos los cultivares son clones. Cuando se habla de "híbridos" de piña, sólo se hace referencia a que el primer representante del clon fue obtenido a través de hibridación sexual entre dos cultivares o materiales experimentales, productos intermedios del mejoramiento genético. Además, sólo la recombinación sexual puede explicar la muy alta diversidad observada entre cultivares de piña precolombinos y la existencia de homocigotos para mutaciones raras, tales como el carácter "piping" en el cultivar Primavera (Cabral et al., 1997).

La mutación somática es una fuente secundaria pero importante de variación, ya que es particularmente frecuente en piña (Collins, 1960); puede aparecer en cualquier parte de una planta, convirtiéndose en una quimera. Retoños provenientes de un sector completamente mutado, son generalmente mutantes estables (Coppens d'Eeckenbrugge, 2014). A excepción de las mutaciones ornamentales, tales como hojas variegadas o frutos de color rojo brillante, la mayoría de las mutaciones cualitativas son desfavorables. Las mutaciones de rasgos cuantitativos pueden progresivamente diferenciar sub-clones de un mismo cultivar. Aunque las variaciones resultantes no sean lo suficientemente claras para justificar su registro como nuevos cultivares, estas selecciones son importantes en términos de rendimiento de campo e ingresos, por lo que pueden justificar el uso de un nombre distinto o código junto al del cultivar original (Coppens d'Eeckenbrugge y Leal, 2003).

Las incoherencias encontradas entre la clasificación morfológica y la diversidad molecular en piña, podrían explicarse por mutaciones similares sobre diferentes fondos genéticos, que dieron lugar a diferentes rasgos morfológicos (Duval et al., 2001).

En el sistema de reproducción de la piña coexisten, un sistema de reproducción sexual alógamo y funcional y un sistema de propagación vegetativa dominante y muy eficiente. La reproducción sexual tiene gran importancia en la variabilidad genética observada en el género Ananas y, en $A$. comosus ésta es esencialmente de origen sexual. La contribución de las mutaciones somáticas puede considerarse importante en la aparición de ciertas características de valor económico, pero es pequeña en comparación con los efectos de la recombinación sexual. A. comosus se considera autoincompatible; la ausencia de semillas en cultivares de esta especie es el resultado de la baja fecundidad y de la autoincompatibilidad (Coppens d'Eeckenbrugge y Duval, 1995).

Para el mejoramiento genético de la piña, es prioritario ampliar su base genética; mediante la evaluación de sus diferentes recursos genéticos, es posible 
identificar caracteres potencialmente útiles (Coppens d'Eeckenbrugge, 1996). La sustitución de cultivares nativos por cultivares mejorados y la acelerada tala de vegetación que viene ocurriendo en las regiones consideradas como centros de diversidad genética de la piña, son las principales causas de erosión genética en el género Ananas. La utilización de pocos cultivares es considerado un problema a ser resuelto por medio de mejoramiento genético y promoviendo la diversificación de los cultivares utilizados para siembra (Cabral et al., 1997).

Expediciones de colecta e introducción de germoplasma de instituciones del Brasil y del exterior, posibilitaron la formación del Banco Activo de Germoplasma de Piña, con 699 accesiones. Además de A. comosus, contiene accesiones de otras especies afines, principalmente de los géneros Ananas y Pseudananas (Cabral et al., 1997). El germoplasma de piña se conserva en varias colecciones de campo alrededor del mundo; las más importantes son las mantenidas por EMBRAPA/ CNPMF en Cruz das Almas, Brazil; la de CIRAD-FLHOR en Martinica y la colección de USDA en Hawaii (Yanes et al., 2005). Los principales países con colecciones de la variabilidad genética de esta especie son: Brasil con 851 accesiones, Estados Unidos 274, Francia 227, Costa de Marfil 129, Japón 109, Nigeria 84, Malasia 54, Taiwán 54, Indonesia 48, Australia 40, la India 33, México 31, España 25, Vietnam 21, Mozambique 21, Tailandia 16, Camerún 14, Nicaragua 14, Filipinas 10 y Costa Rica 5 (Crestani et al., 2010). En Colombia, se reporta una colección de germoplasma con 60 accesiones (Valencia et al., 2010).

El objetivo del presente trabajo fue evaluar la variabilidad genética en ocho accesiones del género Ananas que hacen parte de una colección de trabajo de germoplasma de piña del Centro de Investigación La Libertad de CORPOICA en Villavicencio.

\section{Materiales y métodos}

\section{Localización y descripción del área del estudio}

La colección se encuentra establecida en un suelo ácido de sabana de terraza alta del piedemonte del Meta, en el Centro de Investigación La Libertad de CORPOICA, ubicado en el municipio de Villavicencio, Departamento del Meta, Colombia, en el km 21 de la vía a Puerto López, a $04^{\circ} 03^{\prime} \mathrm{N}, 73^{\circ} 29^{\prime} \mathrm{W}$ y una altitud de $336 \mathrm{msnm}$. El clima se caracteriza por una temperatura promedio anual de $26.3{ }^{\circ} \mathrm{C}$ y una precipitación media de $2916 \mathrm{~mm}$ al año, promedio de 19 años de registros.

\section{Material genético}

Se evaluaron y estimaron descriptores morfológicos cuantitativos y cualitativos en la fase vegetativa de su ciclo, de ocho accesiones: seis de la especie comestible de piña Ananas comosus var. comosus (Híbrido MD-2, Cayena Lisa, Mayanez, Clavo, Perolera y Mitú) y dos genotipos ornamentales A. comosus spp. De estos ocho genotipos, en el plazo de once meses del proyecto, sólo se alcanzaron a medir descriptores tanto de la fase vegetativa como de la fase de fruto, en cuatro genotipos de la especie Ananas comosus var. comosus a saber: el híbrido MD-2, los cultivares comerciales Cayena Lisa y Mayanéz y el cultivar nativo Mitú. A los restantes cuatro genotipos sólo se les alcanzó a medir descriptores de su fase vegetativa.

\section{Diseño experimental}

La toma de los datos de los diferentes descriptores se realizó en muestreo al azar de cinco plantas dentro de cada una de las parcelas establecidas en campo de los diferentes genotipos. Se registraron en la base de datos medidas de 15 descriptores cuantitativos y 27 descriptores cualitativos, tanto vegetativos como de fruto, tomados según recomendaciones del International Plant Genetic Resources Institute - IPGRI, por su sigla en inglés (IPGRI, 1991), hoy Bioversity International.

\section{Análisis estadístico}

A las medidas resultantes de la caracterización de los caracteres cualitativos, se les realizó análisis de frecuencia y se muestran en este trabajo sólo los que presentaron mayores variaciones entre las accesiones evaluadas. Los datos de 15 descriptores cuantitativos evaluados en cuatro de los genotipos, se analizaron mediante prueba de comparación de medias y análisis de componentes principales usando el sistema estadístico SAS v.8e. Antes de aplicar la prueba de comparación de medias, se realizó prueba de normalidad de varianzas con el estadístico Shapiro - Wilk. A las variables que no se ajustaron a la normalidad, se les aplicó transformación de los datos, siendo efectiva la transformación $1 / X$.

\section{Resultados}

\section{Descriptores cualitativos con mayor capacidad discriminante}

De un total de 27 descriptores cualitativos, en la Tabla 2 se muestran los que presentaron mayor capacidad discriminante dentro del grupo de cuatro genotipos 
Tabla 1. Longitud de espinas (L. Esp.), distancia de la punta de la espina al borde de la hoja (D.P.E.B.H) y distribución de espinas en márgenes de hojas en ocho genotipos del género Ananas. Villavicencio. 2013.

\begin{tabular}{|c|c|c|c|c|}
\hline \multirow{2}{*}{ Genotipo } & \multirow{2}{*}{ Rep } & L. Esp. & D.P.E.B.H & \multirow{2}{*}{ Distribución de espinas en hojas } \\
\hline & & $(\mathbf{m m})$ & $(\mathrm{mm})$ & \\
\hline \multirow{5}{*}{ MD-2 } & 1 & 0,84 & 0,63 & \multirow{6}{*}{ Sólo atrás de la punta y cerca de la base } \\
\hline & 2 & 1,38 & 0,86 & \\
\hline & 3 & 1,21 & 0,54 & \\
\hline & 4 & 1,15 & 0,65 & \\
\hline & 5 & 0,97 & 0,71 & \\
\hline Promedio & & 1,11 & 0,68 & \\
\hline \multirow{5}{*}{ Cayena lisa } & 1 & 1,46 & 0,84 & \multirow{6}{*}{ Sólo atrás de la punta y cerca de la base } \\
\hline & 2 & 1,18 & 0,61 & \\
\hline & 3 & 1,83 & 0,8 & \\
\hline & 4 & 1,5 & 0,69 & \\
\hline & 5 & 1,3 & 0,81 & \\
\hline Promedio & & 1,45 & 0,75 & \\
\hline \multirow{5}{*}{ Mayanez } & 1 & 2,71 & 1,33 & \multirow{6}{*}{ A lo largo de todos los márgenes } \\
\hline & 2 & 2,74 & 1,58 & \\
\hline & 3 & 2,61 & 1,63 & \\
\hline & 4 & 2,68 & 1,38 & \\
\hline & 5 & 2,72 & 1,57 & \\
\hline Promedio & & 2,69 & 1,498 & \\
\hline \multirow{5}{*}{ Clavo } & 1 & 0,89 & 0,63 & \multirow{6}{*}{ Irregularmente en ambos márgenes } \\
\hline & 2 & 0,78 & 0,47 & \\
\hline & 3 & 0,97 & 0,61 & \\
\hline & 4 & 1,18 & 0,76 & \\
\hline & 5 & 1,33 & 0,66 & \\
\hline Promedio & & 1,03 & 0,626 & \\
\hline \multirow{5}{*}{$\begin{array}{l}\text { Ornamental } \\
\text { A. comosus spp. }\end{array}$} & 1 & 2,558 & 1,262 & \multirow{6}{*}{ A lo largo de todos los márgenes } \\
\hline & 2 & 2,55 & 1,446 & \\
\hline & 3 & 2,668 & 1,494 & \\
\hline & 4 & 2,684 & 1,284 & \\
\hline & 5 & 2,398 & 1,226 & \\
\hline Promedio & & 2,5716 & 1,3424 & \\
\hline \multirow{5}{*}{$\begin{array}{l}\text { Ornamental roja } \\
\text { A. comosus spp. }\end{array}$} & 1 & 4,31 & 2,88 & \multirow{6}{*}{ A lo largo de todos los márgenes } \\
\hline & 2 & 3,92 & 2,02 & \\
\hline & 3 & 3,74 & 1,95 & \\
\hline & 4 & 3,28 & 1,54 & \\
\hline & 5 & 4,79 & 2,39 & \\
\hline Promedio & & 4,008 & 2,156 & \\
\hline Perolera & & \multicolumn{2}{|c|}{$\begin{array}{l}\text { Hojas "piping" (con } \\
\text { márgenes involutos). }\end{array}$} & Sin espinas en los márgenes \\
\hline Mitú & & \multicolumn{2}{|c|}{$\begin{array}{l}\text { Hojas "piping" (con } \\
\text { márgenes involutos). }\end{array}$} & Sin espinas en los márgenes \\
\hline
\end{tabular}


Tabla 2. Porcentaje de incidencia interespecífica de algunos caracteres cualitativos en cuatro genotipos de piña. Villavicencio. 2013.

\begin{tabular}{|c|c|c|c|}
\hline \multirow[t]{2}{*}{ Descriptor } & \multirow[t]{2}{*}{ Cualidad } & $\begin{array}{c}\text { Incidencia } \\
\text { interespecífica }\end{array}$ & \multirow[t]{2}{*}{ Frecuencia } \\
\hline & & $(\%)$ & \\
\hline \multirow{5}{*}{ Color de la hoja media } & Verdoso/verde (1) & 35 & 7 \\
\hline & Verde con moteado amarillo (2) & 20 & 4 \\
\hline & Verde con manchas rojas (3) & 15 & 3 \\
\hline & Rojo (5) & 5 & 1 \\
\hline & Otro (Margen verde oscuro y centro púrpura) & 25 & 5 \\
\hline \multirow{4}{*}{$\begin{array}{l}\text { Distribución de espinas } \\
\text { en márgenes de hojas }\end{array}$} & Espinas únicamente en la punta (1) & 5 & 1 \\
\hline & Espinas en la punta y cerca de la base (3) & 45 & 9 \\
\hline & Espinas a lo largo de todos los márgenes (4) & 25 & 5 \\
\hline & Otro (Margen involuto, sin espinas) & 25 & 5 \\
\hline \multirow{5}{*}{ Color del pedúnculo } & Verdoso/verde (1) & 30 & 6 \\
\hline & Verde oscuro (2) & 5 & 1 \\
\hline & Verde con moteado amarillo/rojo (3) & 55 & 11 \\
\hline & Rojo (5) & 5 & 1 \\
\hline & Rojo oscuro (6) & 5 & 1 \\
\hline \multirow{3}{*}{ Forma del fruto } & Redonda (3) & 5 & 1 \\
\hline & Cilíndrica (7) & 70 & 14 \\
\hline & Otra (Cilíndrica alargada) & 25 & 5 \\
\hline \multirow{3}{*}{$\begin{array}{l}\text { Espinas en hojas } \\
\text { de la corona }\end{array}$} & Lisas, sin espinas $(1)$ & 25 & 5 \\
\hline & Espinas en la punta (2) & 50 & 10 \\
\hline & Espinosa - aserrada (3) & 25 & 5 \\
\hline
\end{tabular}

evaluados para caracteres vegetativos y de frutos, a saber: MD-2, Cayena Lisa, Mayanez y Mitú. Se observa que se presentó una importante variabilidad en los recursos genéticos respecto de caracteres como: color de la hoja media (que en el cultivar nativo Mitú son de margen verde oscuro y centro púrpura) y del pedúnculo; forma del fruto, especialmente por el fruto del cultivar nativo Mitú que es más alargado que el de los otros genotipos y muy especialmente una alta variabilidad en la distribución de espinas en márgenes de hojas: muy semejante en MD-2 y Cayena Lisa con espinas principalmente en la punta de la hoja y cerca de la base, espinas a lo largo de todos los márgenes en el cultivar Mayanez y, márgenes involutos, sin espinas en el cultivar nativo Mitú; así como variabilidad en el caracter espinas en las hojas de la corona.

La alta variabilidad del caracter distribución de espinas en márgenes de hojas, además de la longitud de espinas y de la distancia de la punta de la espina al borde de la hoja, se puede también apreciar en la Tabla 1, para los ocho genotipos de la Colección de Trabajo de Piña del C.I. La Libertad, a los que se les evaluaron descriptores de la fase vegetativa. Además de la variabilidad ya descrita para los cuatro genotipos anteriores, el cultivar Clavo presentó otra forma de distribución de espinas, siendo estas irregularmente distribuidas en ambos márgenes de las hojas; los dos Genotipos Ornamentales presentaron espinas a lo largo de todos los márgenes y el cultivar Perolera también exhibió el caracter hojas "piping" con márgenes involutos sin espinas.

\section{Análisis de componentes principales (ACP)}

Para el análisis de componentes de varianza en los cuatro genotipos se utilizó la metodología de Kaiser (1960), que consiste en la selección de los componentes cuyo valor propio sea $\geq 1$. Con base en este criterio se seleccionaron los cuatro primeros componentes principales, los cuales explicaron algo más del $83 \%$ de 
la variación total dentro de las 15 variables activas analizadas (Tabla 3).

El primer componente principal contribuyó con el $38.66 \%$, el segundo componente con el $22.98 \%$, el tercer componente con el $15.06 \%$ y el cuarto componente con el $6.67 \%$ de la variación total explicada, de tal manera que los cuatro en conjunto explicaron el 83\% de la variación total (Tabla 3 ).

El primer componente principal contribuyó con casi el $39 \%$ de la varianza total explicada, mientras que la distribución de los coeficientes del primer vector propio indica que: la longitud del pedúnculo (LPED), altura de planta (ALPL), longitud del fruto (LFR) y ancho de la hoja $\mathrm{D}(\mathrm{AHD})$, fueron las variables que más contribuyeron en forma positiva a dicho componente. De forma secundaria lo hicieron acidez (ACID) y longitud de la hoja D (LHD). Por otro lado, las variable grados Brix (BRIX) o sólidos solubles totales, espesor medio de la hoja D (EMHD) y diámetro del pedúnculo (DPED), fueron las que más contribuyeron en forma negativa a este primer componente (Tabla 4).

Los resultados del primer componente indican que éste permitió diferenciar los genotipos con un alto vigor vegetativo representado en ALPL mayor, hoja D ancha y larga, pedúnculo largo y un fruto con mayor longitud y acidez que el de los otros genotipos evalua- dos, como es el caso principalmente del cultivar nativo Mitú y del cultivar Cayena Lisa. También coincide la contribución en forma negativa de la variable BRIX, pues Mitú presentó la menor concentración (13.7) de sólidos solubles totales, inferior significativamente a MD-2 y a Mayanez y sin diferencia de Cayena Lisa (Tabla 5). También coincide el hecho que el cultivar nativo Mitú, además de presentar un pedúnculo largo es también de bajo diámetro, lo que en campo produce alguna susceptibilidad a caída de frutos y sus hojas D son largas y anchas pero de bajo espesor medio (Tabla 5).

Para el segundo componente principal, las variables con mayor efecto positivo fueron el diámetro medio del fruto (DMF), el diámetro del corazón (DCOR) y el peso del fruto (PFR), (Tabla 4). En conclusión, con este segundo componente fue posible distinguir los genotipos con mayor tamaño y PFR, en este caso especialmente el genotipo Cayena Lisa (Tabla 5).

En el análisis del tercer componente, la variable que más contribuyó en sentido positivo con el mismo, fue el peso de la planta (PPL), seguido del peso de la hoja D (PHD) (Tabla 4), permitiendo distinguir genotipos con estas dos características, especialmente el genotipo Mayanez el cual presentó los mayores PPL y PHD (Tabla 5).

Tabla 3. Valores propios y proporción de la varianza total explicada en el análisis de componentes principales.

\begin{tabular}{|c|c|c|c|}
\hline \multirow{2}{*}{$\begin{array}{c}\text { Componentes } \\
\text { principales }\end{array}$} & \multirow{2}{*}{$\begin{array}{l}\text { Valores } \\
\text { propios }\end{array}$} & Proporción de la varianza total explicada \\
\cline { 3 - 4 } & 57.986 & 38.66 & Acumulada \\
\hline 1 & 34.465 & 22.98 & 38.66 \\
\hline 2 & 22.587 & 15.06 & 61.63 \\
\hline 3 & 10.002 & 6.67 & 76.69 \\
\hline 4 & 0.7489 & 4.99 & 83.36 \\
\hline 5 & 0.5047 & 3.37 & 88.35 \\
\hline 6 & 0.3789 & 2.53 & 91.72 \\
\hline 7 & 0.2918 & 1.95 & 94.25 \\
\hline 8 & 0.1779 & 1.19 & 96.19 \\
\hline 9 & 0.1561 & 1.04 & 97.38 \\
\hline 10 & 0.0997 & 0.66 & 98.42 \\
\hline 11 & 0.0628 & 0.42 & 99.08 \\
\hline 12 & 0.0403 & 0.27 & 99.50 \\
\hline 13 & 0.0290 & 0.19 & 99.77 \\
\hline 14 & 0.0050 & 0.03 & 99.97 \\
\hline 15 & & & 100.00 \\
\hline
\end{tabular}


Tabla 4. Coeficientes propios para los cuatro primeros componentes principales.

\begin{tabular}{|l|r|r|r|r|}
\hline \multirow{2}{*}{ Características } & \multicolumn{4}{c|}{ Componentes Principales } \\
\cline { 2 - 5 } & $\mathbf{1}$ & \multicolumn{1}{c|}{$\mathbf{2}$} & \multicolumn{1}{c|}{$\mathbf{3}$} & \multicolumn{1}{c|}{$\mathbf{4}$} \\
\hline Altura de planta & 0.36 & -0.03 & 0.01 & 0.18 \\
\hline Peso de planta & 0.04 & 0.21 & 0.55 & -0.04 \\
\hline Número de hojas & 0.04 & 0.3 & 0.03 & -0.65 \\
\hline Longitud hoja D & 0.28 & -0.28 & 0.3 & 0.03 \\
\hline Ancho hoja D & 0.34 & 0.08 & 0.09 & 0.31 \\
\hline Espesor medio Hoja D & -0.28 & -0.09 & 0.13 & 0.43 \\
\hline Peso hoja D & -0.11 & -0.24 & 0.43 & -0.29 \\
\hline Longitud del pedúnculo & 0.38 & -0.11 & 0.03 & -0.13 \\
\hline Diámetro del pedúnculo & -0.24 & 0.28 & 0.3 & 0.15 \\
\hline Peso del fruto & 0.13 & 0.35 & 0.33 & 0.01 \\
\hline Longitud del fruto & 0.34 & -0.06 & 0.18 & 0.21 \\
\hline Diámetro medio del fruto & -0.01 & 0.49 & 0.07 & 0.1 \\
\hline Diámetro del corazón & -0.04 & 0.43 & -0.2 & 0.14 \\
\hline Brix & -0.35 & -0.08 & 0.1 & 0.17 \\
\hline Acidez & 0.3 & 0.2 & -0.27 & 0.04 \\
\hline
\end{tabular}

En el cuarto componente principal, la variable que más contribuyó en sentido negativo fue el número de hojas (NHO) y en sentido positivo el EMHD (Tabla 4), permitiendo distinguir genotipos con un bajo número de hojas y con un alto espesor medio de la hoja D, como fue el caso del híbrido MD-2 que presentó el mayor espesor con $2.68 \mathrm{~mm}$, diferente significativamente de los otros tres genotipos (Tabla 5).

De tal manera que, de los descriptores vegetativos cuantitativos: ALPL; los descriptores de la hoja D: AHD y PHD y de los descriptores de fruto: LPED y LFR, fueron los que presentaron mayor variabilidad en el grupo de accesiones de piña evaluadas y por lo tanto, los más discriminantes dentro del Análisis de de Componentes Principales. Mientras que, en cuanto a los descriptores cualitativos, la mayor variación dentro del germoplasma caracterizado, se observó en el color de la hoja media y del pedúnculo, la forma del fruto, la longitud de las espinas y muy especialmente una alta variabilidad en la forma de distribución de las espinas en los márgenes de hojas.

Con base en los promedios de descriptores morfológicos cuantitativos de la Tabla 5 y en las características de espinas mostradas en la Tabla 1, se pueden resumir las principales características que mostraron en esta evaluación los cuatro genotipos de piña a los que se les evaluaron descriptores vegetativos y de fruto:
El cultivar nativo Mitú se caracterizó principalmente por: una mayor longitud y anchura de la hoja D; una alta longitud y bajo diámetro del pedúnculo que favorece en campo alguna caída de frutos y una significativa mayor longitud del fruto. Sus márgenes de hojas son tipo "piping", sin espinas y con margen involuto, aunque menos evidente que en el cultivar Perolera, sí se observa en plantas adultas y vigorosas creciendo en suelos fértiles, debido a que el margen involuto es menos prominente, más plano y de un tono verde ligeramente claro y menos blanco que en Perolera. En plantas jóvenes creciendo en un suelo de sabana de la Universidad de los Llanos en Villavicencio, fue más evidente el carácter "piping" de sus márgenes de hojas. Fue el genotipo más disímil a los otros evaluados y debe seguir siendo observado como potencial donante, en búsqueda de otros genes útiles para el mejoramiento genético.

Cayena Lisa se caracterizó principalmente por: el mayor número de hojas; el menor peso y espesor medio de la hoja D y el mayor peso del fruto y acidez. Presentó espinas de $1.45 \mathrm{~mm}$ en promedio, sólo atrás de la punta y cerca de la base.

El genotipo Mayanez se caracterizó principalmente por: el mayor peso de la hoja D; grados Brix un poco menor pero sin diferencia significativa con el híbrido MD-2 y baja acidez del fruto. Sus espinas más largas, dentro de las accesiones cultivadas comercialmente, 


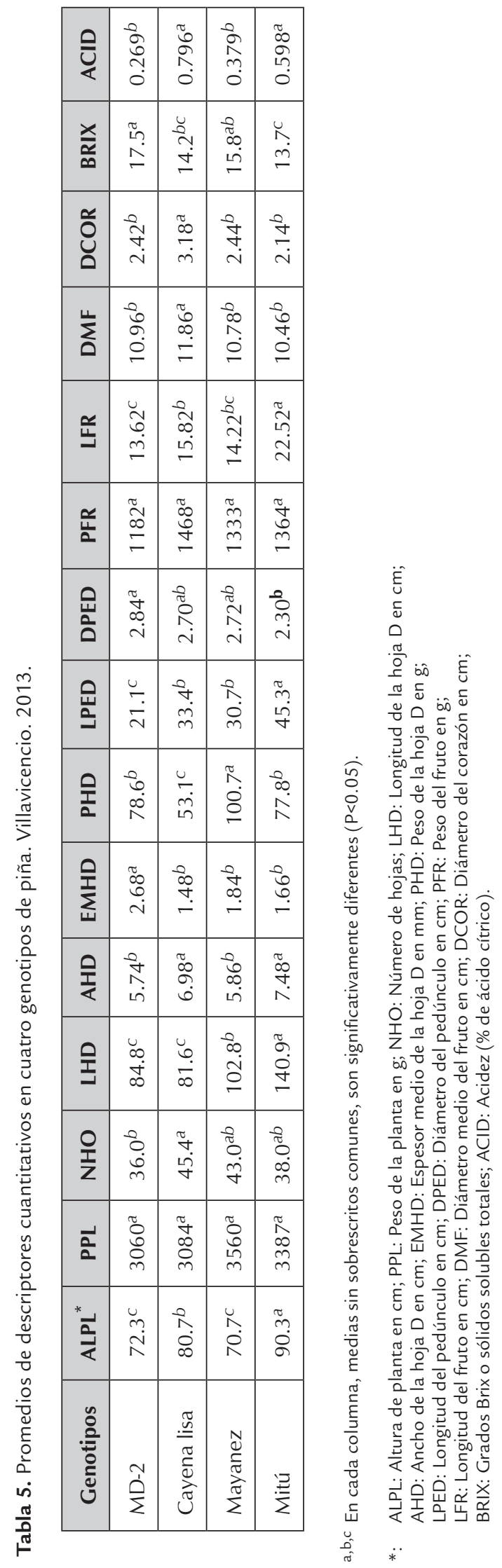


con $2.69 \mathrm{~mm}$ en promedio y distribuidas a lo largo de todos los márgenes de sus hojas. En visita a fincas de productores de este cultivar en Paratebueno (Cundinamarca), ellos manifiestan que sería conveniente, mediante mejoramiento genético, lograr un nuevo cultivar, con las calidades organolépticas de Mayanez, pero sin espinas en sus márgenes de hojas.

El híbrido mejorado MD-2 se caracterizó principalmente por: una baja altura de planta y número de hojas; el significativamente mayor espesor medio de la hoja D; la significativamente más corta longitud del pedúnculo; el mayor diámetro del pedúnculo; el menor peso y longitud del fruto; los más altos grados Brix, diferentes significativamente de Cayena Lisa y de Mitú (Tabla 5); la menor acidez del fruto y la presencia de espinas cortas de $1.11 \mathrm{~mm}$ en promedio, sólo atrás de la punta y cerca de la base de las hojas.

\section{Discusión}

Los resultados obtenidos en esta investigación para el cultivar Cayena Lisa (Tabla 5), coinciden con los reportes de otros autores como Sampaio et al., (2011), quienes en Brasil también observaron producción de frutos de mayor tamaño y peso de Cayena lisa frente a otros genotipos. Además, su mayor acidez en frutos, frente a otro cultivar, Pérola, fue también reportada (Cunha y Rocha, 1993; Barreiro Neto et al., 1998).

Durante mucho tiempo, Cayena Lisa representó prácticamente la única variedad para exportación en fresco y enlatada. Sin embargo, el híbrido MD-2 le ha quitado su hegemonía en el mercado, principalmente debido a su extraordinaria capacidad para soportar el transporte en frío. Esta resistencia en poscosecha era desconocida y abre nuevas perspectivas para la obtención de nuevos cultivares mejorados por hibridación. En los mercados locales, se prefieren otras variedades con interesantes cualidades gustativas, pero con muy baja capacidad de almacenamiento, como Pérola en Brasil, Reina en Asia o el Océano Índico (Denis y Thierry, 2012) y el caso del cultivar Mayanez, en los Llanos Orientales de Colombia.

Según Cabral (2000), entre algunas de las características deseables en una variedad mejorada de piña se encuentran: crecimiento rápido; hojas cortas, anchas y sin espinas; cáscara del fruto amarillo-anaranjada; ojos planos; pulpa amarilla, firme más no fibrosa; contenido de azúcar elevado; acidez moderada y corona media a pequeña. Además de alto rendimiento y con resistencia o tolerancia a las principales plagas y enfermedades. Sin embargo, como es difícil encontrar una variedad que reúna todas las características ideales, es conveniente escoger variedades para usos específicos según las demandas de los consumidores y con adaptación específica a las regiones de siembra. Con relación a la longitud del pedúnculo, un buen cultivar de piña debe presentar valores menores de $20 \mathrm{~cm}$, para evitar la caída de frutos y el golpe de sol (Cabral et al., 2009). En el presente trabajo, el híbrido MD-2 presentó los datos de pedúnculo más corto con 21.1 $\mathrm{cm}$, diferente significativamente de los otros tres cultivares evaluados para características de frutos, además de su mayor contenido de azúcar de 17.5 grados Brix (Tabla 5).

En Brasil, las normas de clasificación de piña consideran que el peso del fruto comercial debe estar entre 900 y 2400 g, el contenido de azúcares debe ser superior de $12^{\circ}$ Brix. Para la industrialización de la piña, el contenido de azúcares debe estar entre 14 y $16^{\circ}$ Brix, y la acidez total entre 0.65 y 0.95 (Cabral et al., 2009). Esto indica que los cuatro genotipos evaluados para características de fruto, cumplen todos el requisito de grados Brix para consumo en fresco, pero para industrialización, el cultivar nativo Mitú se mostró ligeramente abajo con 13.7 (Tabla 5).

En la presente investigación, no obstante que el número de genotipos evaluados fue bajo, se encontró una alta variabilidad en la forma de distribución de las espinas en los márgenes de hojas. Según Coppens d'Eeckenbrugge y Sanewski (2011), la forma del margen de la hoja es un importante descriptor en piña y es una de las características distintivas de los cultivares y variedades botánicas. Más importante aún, puede ser un elemento diferenciador clave para la protección de la propiedad intelectual, como los derechos de obtentor o patentes de plantas.

Ya originalmente, Collins (1960) describió seis tipos de márgenes foliares, a saber: espinosa, punta espinosa, festón o recorte curvado, liso, ribete (piping) y papel de lija. Posteriormente, Loison-Cabot (1990) describió también seis tipos, pero, considerando las grandes espinas de Ananas comosus var. bracteatus como un tipo distinto y omitiendo el tipo recorte curvado. Más recientemente, Coppens d'Eeckenbrugge y Sanewski (2011), a sabiendas que el medio ambiente puede influir en la expresión de las espinas en algunos genotipos base y esto, junto con un cierto malentendido, puede dar lugar a descripciones erróneas de variedades, describen seis tipos de márgenes de hojas con utilidad principal para efecto de descripción varietal, así: espinosa, espinosidad parcial, punta espinosa, ribete (piping), lisa y papel de lija. Omiten el tipo festón o recorte curvado, dado que Collins (1960) da poca 
descripción de él y el fenotipo no se ha observado por dichos autores.

El margen de la hoja en ribete o "piping" es usualmente explicado como un margen de hoja plegado que es visible a simple vista como un margen blanco/plateado. Por lo general, se atribuye a una tira delgada de la superficie epidérmica inferior de la hoja que se extiende sobre la superficie superior (Coppens d'Eeckenbrugge y Sanewski, 2011). El margen blanco surge de una mayor densidad de tricomas (Collins y Kerns, 1946) y posiblemente un color epidermal más claro. Entretanto, el margen liso de la hoja no es común en cultivares comerciales para consumo en fresco o procesamiento. La forma botánica A. comosus var. erectifolious, incluyendo el clon ornamental "Selvagem 6", se caracterizan por un margen liso de la hoja, sin un margen superior blanco o plegado. Unas pocas espinas aisladas son a veces observadas, en particular hacia la base de la hoja, pero no en la punta de la hoja (Coppens d'Eeckenbrugge y Sanewski, 2011).

En la presente investigación de descripción de la colección de trabajo de piña del C.I. La Libertad, se encontraron genotipos representantes de cuatro de los seis tipos de márgenes de hojas descritos por Coppens d'Eeckenbrugge y Sanewski (2011), así: espinosa (en el caso del cultivar Mayanez y los dos genotipos ornamentales); espinosidad parcial, en el caso del cultivar Clavo; punta espinosa, en el híbrido MD-2 y el cultivar Cayena Lisa y ribete u hoja "piping" en el cultivar comercial Perolera y en el cultivar nativo Mitú (Tabla 1 ).

Además del tipo de borde de hoja "piping" del cultivar nativo Mitú, más deseable para manejo comercial que los bordes espinosos, éste presentó también la máxima longitud del fruto con $28.3 \mathrm{~cm}$ y el mayor promedio con $22.52 \mathrm{~cm}$ (Tabla 5), diferente significativamente respecto al híbrido MD-2, Cayena Lisa y Mayanez. Loison-Cabot (1990), consideró a este carácter, al que llamó "fruto elongado", como una mutación ventajosa y un rasgo hereditario interesante para la obtención de nuevas variedades.

Estas características del cultivar nativo Mitú, hacen de él un recurso genético valioso como donante de algunos caracteres deseables para el mejoramiento genético, y ponen de manifiesto la importancia de continuar colectando, conservando y caracterizando los recursos genéticos nativos de la piña existentes en la Orinoquia, la Amazonia y el resto del país, antes que los mismos desaparezcan, sin ser aprovechados adecuadamente, por efecto de la co- lonización, la tala de bosques, la sustitución de cultivares nativos por cultivares mejorados y los efectos adversos del cambio climático sobre los recursos fitogenéticos.

El tipo de borde de hoja "piping" que caracteriza especialmente al cultivar Perolera de A. comosus, pero es posible encontrarlo también en algunos tipos espontáneos de la Amazonia venezolana (Loison-Cabot, 1990); pero también a lo largo del alto Amazonas, cerca de la triple frontera entre Colombia, Perú y Brasil y en el bajo Río Negro, donde dicho carácter es relativamente frecuente (Coppens d'Eeckenbrugge y Duval, 2009). Tal es el caso del cultivar nativo Mitú evaluado en el presente trabajo y que seguramente fue traído por productores, desde áreas del Departamento del Vaupés, Colombia

La utilización del germoplasma silvestre y primitivo es prometedora para el mejoramiento genético de la piña; sin embargo, está limitada por la difícil caracterización e introgresión de los rasgos de resistencia. En comparación, el desarrollo de cultivares ornamentales parece sencillo, ya que los rasgos interesantes son mucho más fáciles de manipular. Otros usos, como la producción de fibra de alta calidad y los usos para tratamientos farmacéuticos, abren nuevas perspectivas comerciales. Las colecciones de germoplasma de Ananas, claramente necesitan más investigaciones para desarrollar su pleno potencial (Coopens d'Eeckenbrugge et al., 2011).

No obstante que la colección de trabajo evaluada fue pequeña, se cuenta con variabilidad genética útil y es muy importante, mediante colectas e intercambio con pequeños productores, continuar la ampliación de la misma con la búsqueda de otros cultivares nativos, su adecuada caracterización y evaluación en las condiciones locales de los Llanos para que sean útiles como donantes de genes para proyectos de mejoramiento genético y para uso directo, de algunos de ellos, en pequeña producción orgánica para consumo en fresco. Lo anterior, dado que, según FAO (2011), el cambio climático representa grandes amenazas a los recursos fitogenéticos para la alimentación y la agricultura, y por tanto, a nuestra seguridad alimentaria y como principal garantía para poder enfrentarlo, requeriremos acceder a una amplia disponibilidad de recursos fitogenéticos, conservar, caracterizar y utilizar las variedades nativas y especies silvestres relacionadas con el cultivo y toda la variabilidad genética existente en las colecciones de germoplasma, como fuentes de genes de adaptación para resistencia biótica y abiótica al cambio climático. 


\section{Agradecimientos}

Los autores agradecen al Instituto de Investigaciones de la Orinoquia Colombiana IIOC de la Universidad de los Llanos por el apoyo financiero al proyecto. Igualmente a CORPOICA por su colaboración al facilitar la realización de las evaluaciones y en esta entidad a: la l. A. Claudia Liliana Calderón y a los auxiliares de campo Abraham Ramirez y Rodrigo Prieto. En la Universidad de los Llanos a la estudiante de Ingeniería Agronómica Juliette Riaño por su colaboración en la toma de descriptores y al Profesor Jorge Enrique Muñoz por sus valiosos aportes y conocimiento del manejo agronómico del cultivo de la piña, como profesional y productor.

\section{Referencias}

Botella JR, Smith M. 2008. Genomics of Pineapple, Crowning the King of Tropical Fruits. In: Moore, PH, Ming, R (Editors). Plant Genetics/Genomics: Genomics of Tropical Crop Plants. United States: Springer. pp. 441- 451.

Barreiro NM, Choairy SA, Lacerda JT, Carvalho RA, Oliveira EF. Caracterização do abacaxizeiro Pérola no Estado da Paraíba. Pesquisa Agropecuária. 1998;10:33-39.

Cabral JRS, Souza JDS, Ferreira FR. 1997. Variabilidade genética e melhoramento do abacaxi. Recursos genéticos e melhoramento de plantas para o nordeste brasileiro. [Disponível el 31/08/2013 en URL: <http://www.cpatsa.embrapa.br:8080/ catalogo/livrorg/abacaxi.pdf>].

Cabral JRS. 2000. Variedades. In: Reinhardt DH et al. (Org.). Abacaxi produção: aspectos técnicos. Brasília: Embrapa. pp. 15 - 18.

Cabral JRS, Ledo CAD, Caldas RC, Junghans DT. Variação de caracteres em híbridos de abacaxizeiro obtidos de diferentes cruzamentos. Rev Bras Frutic. 2009;31(4):1129-1134.

Clement CR, Cristo-Araujo M de, Coppens d'Eeckenbrugge G, Pereira AA, Picanço-Rodrigues D. 2010. Origin and Domestication of Native Amazonian Crops. Diversity. 2010; 2:72-106. doi:10.3390/d2010072

Collins JL, Kerns KR. Inheritance of three leaf types in the pineapple. Journal Heredity. 1946;37:123-128.

Collins JL. 1960. The Pineapple: Botany, Cultivation and Utilization. Interscience Publishers. New York. 294 p.

Coppens d'Eeckenbrugge G, Duval MF. Bases genéticas para definir una estrategia de mejoramiento de la piña. Revista Fac Agron (Maracay). 1995;21:95-118.

Coppens d'Eeckenbrugge G. Pineapple News From the Martinique Symposium. A Brief Overview on Pineapple Breeding Work from the Communications Presented at the Second Pineapple Symposium. Pinneaple News. 1996;2(2):13-16.

Coppens d'Eeckenbrugge G, Leal F. 2003. Morphology, anatomy and taxonomy. In: Bartholomew DP et al. The pineapple - botany, production and uses. Oxon: CABI. pp. $13-32$.
Coppens d'Eeckenbrugge G, Duval MF. The Domestication of Pineapple: Context and Hypotheses. Pinneaple News. 2009; 16:15-27.

Coppens d'Eeckenbrugge G., Sanewski G. Leaf Margin in Pineapple. Pinneaple News. Newsletter of the Pineapple Working Group, International Society for Horticultural Science. ISHS. 2011;18: 32-37.

Coppens D'Eeckenbrugge G, Sanewski GM, Smith MK, Duval MF, Leal F. 2011. Ananas. In: Wild crop relatives: genomic and breeding resources. Tropical and subtropical fruits. Kole Chittaranjan (Editor). Heidelberg: Springer [Allemagne], pp. 21-41. [Disponível el 16/01/2016 en URL: <http://dx.doi.org/10.1007/978-3642-20447-0_2>].

Coppens D'Eeckenbrugge G. Pinneaple taxonomy: Species, botanical varieties and cultivars and their importance in understanding and managing pineapple diversity. Pineapple News. 2014;21:34-38. [Disponível el 16/01/2016 en URL: http:// www.ishs-horticulture.org/workinggroups/pineapple/PineNews21.pdf.].

Crestani M, Barbieri RL, Hawerroth FJ, Carvalho FIF de, Oliveira, AC de. Das Américas para o Mundo - origem, domesticação e dispersão do abacaxizeiro. Ciência Rural, Santa Maria. 2010;40(6):1473-1483.

Cunha GAP, Rocha SL. 1993. Comportamento de cultivares de abacaxi sob irrigação no Norte de Minas Gerais. Cruz das Almas: Embrapa - CNPMF. 2 p. Abacaxi em foco, 95.

Denis L, Thierry P. 2012. Les principales variétés d'ananas. Fruitrop (Édition Française) 204: p. 59. [Disponible el 14/01/2016 en URL: <http://agritrop.cirad.fr/566389/>].

Duval MF, Coppens d'Eeckenbrugge G, Ferreira FR, Cabral JRS, Bianchetti $L$ de $B$. First results from joint EMBRAPA-CIRAD Ananas germplasm collecting in Brazil and French Guyana. Acta Hortic. 1997;425:137-144.

Duval MF, Noyer JL, Hamon P, Coppens d'Eeckenbrugge G. Study of variability in the genera Ananas and Pseudoananas using RFLP. Acta Hortic. 2000;529:123-131.

Duval MF, Noyer JL, Perrier X, Coppens d'Eeckenbrugge G, Hamon P. Molecular diversity in pineapple assessed by RFLP markers. Theor. Appl. Gen. 2001;102(1):83-90.

FAO - Organización de las Naciones Unidas para la Agricultura y la Alimentación. 2011. Cambio climático y recursos genéticos para la alimentación y la agricultura. p. 13. Comisión de Recursos Genéticos para la alimentación y la Agricultura. 13ª Reunión Ordinaria. Roma. [Disponible el 20/01/2016 en URL: http://www.fao.org/docrep/meeting/022/mb335s.pdf].

IPGRI - International Plant Genetic Resource Institute. 1991. Descriptors for Pineapple. Londres: CAB International.

Kaiser HF. The application of electronic computers to factor analysis. Educational and Psychological Measurement. 1960;20:141151.

Loison-Cabot C. Génétique de I"ananas: hérédité de certains caractères, leur stabilité au cours des cycles végétatifs. Fruits. 1990;45(5):447- 455 
Noyer JL, Launad C, Coppens d'Eeckenbrugge G, Duval MF. RFLP study on rDNA in Ananas genus. Acta Horticulturae. 1997;425:153-160.

Py C. 1970. La piña tropical. Editorial Blume. Madrid. 568 p.

Py C, Lacoeuilhe JJ, Teison C. 1984. L'ananas, sa culture, sés produits. Paris: G.P. Maisonneuve et Larose et ACCT. 562 p.

Sampaio AC, Fumis, T de F, Leonel S. Crescimento vegetativo e características dos frutos de cinco cultivares de Abacaxi na região de Bauru-SP. Rev Bras Frutic. 2011;33(3):816-822.

Tapia E, Guillén H, Gutierrez MA. Caraterización genética de materiales de piña (Ananas spp.) mediante RAPD e ISSR. Rev Fitotec Mex. 2005;28(3):187-194.
Valencia RA, Lobo AM, Ligarreto MGA. Estado del arte de los recursos genéticos vegetales en Colombia: Sistema de Bancos de Germoplasma. Revista Corpoica -Ciencia y Tecnología Agropecuaria. 2010;11(1):85-94.

Yanes E, Gil K, Rebolledo L, Martinez O, Isidrón M, Simpson J. AFLP characterization of the mexican pineapple germplasm collection. J Amer Soc Hort Sci. 2005;130(4):575-579.

Zhang J, Liu J, Ming, R. Genomic analyses of the CAM plant pineapple. J Exp Bot. 2014;65(13):3395-3404 